Article

\title{
Construction of Periodic Wavelet Frames Generated by the Walsh Polynomials
}

\author{
Sunita Goyal ${ }^{1}$ and Firdous A. Shah ${ }^{2, *}$ \\ ${ }^{1}$ Department of Mathematics, Shri Jagdishprasad Jhabarmal Tibrewala University (JJTU), \\ Jhunjhunu 333001, Rajasthan, India; E-Mail: sunitagoel2011@gmail.com \\ ${ }^{2}$ Department of Mathematics, University of Kashmir, South Campus, Anantnag 192101, \\ Jammu and Kashmir, India \\ * Author to whom correspondence should be addressed; E-Mail: fashahku@gmail.com; \\ Tel.: +91-1932-227764.
}

Academic Editor: Palle E. T. Jorgensen

Received: 24 August 2015 / Accepted: 20 November 2015 / Published: 3 December 2015

\begin{abstract}
An explicit method for the construction of a tight wavelet frame generated by the Walsh polynomials with the help of extension principles was presented by Shah (Shah, 2013). In this article, we extend the notion of wavelet frames to periodic wavelet frames generated by the Walsh polynomials on $\mathbb{R}^{+}$by using extension principles. We first show that under some mild conditions, the periodization of any wavelet frame constructed by the unitary extension principle is still a periodic wavelet frame on $\mathbb{R}^{+}$. Then, we construct a pair of dual periodic wavelet frames generated by the Walsh polynomials on $\mathbb{R}^{+}$using the machinery of the mixed extension principle and Walsh-Fourier transforms.
\end{abstract}

Keywords: periodic wavelet frame; extension principle; Walsh polynomial; Walsh-Fourier transform

MSC classifications: 42C40; 42C15; 42A38; 41A17; 22B99

\section{Introduction}

Wavelet frames have gained considerable popularity during the past decade, primarily due to their substantiated applications in diverse and widespread fields of engineering and science. One of the 
most useful methods to construct wavelet frames is through the concept of the unitary extension principle (UEP) introduced by Ron and Shen [1] and was subsequently extended by Daubechies et al. [2] in the form of the oblique extension principle (OEP). They give sufficient conditions for constructing tight wavelet frames for any refinable function $\phi(x)$ that generates a multiresolution analysis. The resulting wavelet frames are based on multiresolution analysis, and the generators are often called framelets. These methods of construction of wavelet frames are generalized from one dimension to higher dimensions, tight frames to dual frames, from a single scaling function to a scaling function vector. More importantly, the setup of tight wavelet frames provides great flexibility in approximating and representing periodic functions. Using periodization techniques, Zhang [3] constructed a dual pair of periodic wavelet frames for $L^{2}[0,1]$ under the assumption that the support of the wavelet function $\psi$ in the frequency domain is contained in $[-\pi,-\varepsilon] \cup[\varepsilon, \pi], \varepsilon>0$. Later on, Zhang and Saito [4] constructed general periodic wavelet frames using extension principles. More precisely, they proved that under some decay conditions, the periodization of any wavelet frame constructed by the unitary extension principle is a periodic wavelet frame, and the periodization of any pair of dual wavelet frames constructed by the mixed extension principle is a pair of dual periodic wavelet frames. To mention only a few references on wavelet frames, the reader is referred to [5-8] and the many references therein.

The past decade has also witnessed a tremendous interest in the problem of constructing compactly-supported orthonormal scaling functions and wavelets with an arbitrary dilation factor $p \geq 2, p \in \mathbb{N}$ (see Debnath and Shah [9]). The motivation comes partly from signal processing and numerical applications, where such wavelets are useful in image compression and feature extraction, because of their small support and multifractal structure. Lang [10] constructed several examples of compactly-supported wavelets for the Cantor dyadic group by following the procedure of Daubechies [11] via scaling filters, and these wavelets turn out to be certain lacunary Walsh series on the real line. Kozyrev [12] found a compactly-supported $p$-adic wavelet basis for $L^{2}\left(\mathbb{Q}_{p}\right)$, which is an analog of the Haar basis. The concept of multiresolution analysis on a positive half-line $\mathbb{R}^{+}$was recently introduced by Farkov [13]. He pointed out a method for constructing compactly-supported orthogonal $p$-wavelets related to the Walsh functions and proved necessary and sufficient conditions for scaling filters with $p^{n}$ many terms $(p, n \geq 2)$ to generate a $p$-MRAin $L^{2}\left(\mathbb{R}^{+}\right)$. Subsequently, dyadic wavelet frames on the positive half-line $\mathbb{R}^{+}$were constructed by Shah and Debnath in [14] using the machinery of Walsh-Fourier transforms. They have established necessary and sufficient conditions for the system $\left\{\psi_{j, k}(x)=2^{j / 2} \psi\left(2^{j} x \ominus k\right): j \in \mathbb{Z}, k \in \mathbb{Z}^{+}\right\}$to be a frame for $L^{2}\left(\mathbb{R}^{+}\right)$. Wavelet packets and wavelet frame packets related to the Walsh polynomials were deeply investigated by Shah and Debnath in $[14,15]$. Recent results in this direction can also be found in $[16,17]$ and the references therein.

Recently, Shah [18] established a unitary extension principle for constructing normalized tight wavelet frames generated by the Walsh polynomials on $\mathbb{R}^{+}$. Drawing inspiration from these wavelet frames, our aim is to extend the notion of wavelet frames to periodic wavelet frames on $\mathbb{R}^{+}$by using extension principles. More precisely, we prove that under some mild conditions, the periodization of any wavelet frame constructed by the unitary extension principle is a periodic wavelet frame on a positive half-line $\mathbb{R}^{+}$. Furthermore, based on the mixed extension principle and Walsh-Fourier transforms of the wavelet frames, an explicitly-constructed method for a pair of dual periodic wavelet frames generated by the Walsh polynomials is also given. 
This paper is organized as follows. In Section 2, we introduce some notations and preliminaries related to the operations on positive half-line $\mathbb{R}^{+}$, including the definitions of the Walsh-Fourier transform and MRA-based wavelet frames related to the Walsh polynomials. Sections 3 and 4 state and prove our main results about periodic wavelet frames generated by the Walsh polynomials.

\section{Walsh-Fourier Analysis and MRA-Based Wavelet Frames}

We start this section with certain results on Walsh-Fourier analysis. We present a brief review of generalized Walsh functions, Walsh-Fourier transforms and their various properties.

As usual, let $\mathbb{R}^{+}=[0,+\infty), \mathbb{Z}^{+}=\{0,1,2, \ldots\}$ and $\mathbb{N}=\mathbb{Z}^{+}-\{0\}$. Denote by $[x]$ the integer part of $x$. Let $p$ be a fixed natural number greater than one. For $x \in \mathbb{R}^{+}$and any positive integer $j$, we set:

$$
x_{j}=\left[p^{j} x\right](\bmod p), \quad x_{-j}=\left[p^{1-j} x\right](\bmod p)
$$

where $x_{j}, x_{-j} \in\{0,1, \ldots, p-1\}$. Clearly, $x_{j}$ and $x_{-j}$ are the digits in the $p$-expansion of $x$ :

$$
x=\sum_{j<0} x_{-j} p^{-j-1}+\sum_{j>0} x_{j} p^{-j}
$$

Moreover, the first sum on the right is always finite. Besides,

$$
[x]=\sum_{j<0} x_{-j} p^{-j-1}, \quad\{x\}=\sum_{j>0} x_{j} p^{-j}
$$

where $[x]$ and $\{x\}$ are, respectively, the integral and fractional parts of $x$.

Consider on $\mathbb{R}^{+}$the addition defined as follows:

$$
x \oplus y=\sum_{j<0} \zeta_{j} p^{-j-1}+\sum_{j>0} \zeta_{j} p^{-j}
$$

with $\zeta_{j}=x_{j}+y_{j}(\bmod p), j \in \mathbb{Z} \backslash\{0\}$, where $\zeta_{j} \in\{0,1, \ldots, p-1\}$ and $x_{j}, y_{j}$ are calculated by Equation (1). Clearly, $[x \oplus y]=[x] \oplus[y]$ and $\{x \oplus y\}=\{x\} \oplus\{y\}$. As usual, we write $z=x \ominus y$ if $z \oplus y=x$, where $\ominus$ denotes subtraction modulo $p$ in $\mathbb{R}^{+}$.

Let $\varepsilon_{p}=\exp (2 \pi i / p)$; we define a function $r_{0}(x)$ on $[0,1)$ by:

$$
r_{0}(x)= \begin{cases}1, & \text { if } x \in[0,1 / p) \\ \varepsilon_{p}^{\ell}, & \text { if } x \in\left[\ell p^{-1},(\ell+1) p^{-1}\right), \quad \ell=1,2, \ldots, p-1\end{cases}
$$

The extension of the function $r_{0}$ to $\mathbb{R}^{+}$is given by the equality $r_{0}(x+1)=r_{0}(x), \forall x \in \mathbb{R}^{+}$. Then, the system of generalized Walsh functions $\left\{w_{m}(x): m \in \mathbb{Z}^{+}\right\}$on $[0,1)$ is defined by:

$$
w_{0}(x) \equiv 1 \quad \text { and } \quad w_{m}(x)=\prod_{j=0}^{k}\left(r_{0}\left(p^{j} x\right)\right)^{\mu_{j}}
$$

where $m=\sum_{j=0}^{k} \mu_{j} p^{j}, \mu_{j} \in\{0,1, \ldots, p-1\}, \mu_{k} \neq 0$. They have many properties similar to those of the Haar functions and trigonometric series and form a complete orthogonal system. Further, by a Walsh polynomial, we shall mean a finite linear combination of Walsh functions. 
For $x, y \in \mathbb{R}^{+}$, let:

$$
\chi(x, y)=\exp \left(\frac{2 \pi i}{p} \sum_{j=1}^{\infty}\left(x_{j} y_{-j}+x_{-j} y_{j}\right)\right)
$$

where $x_{j}, y_{j}$ are given by Equation (1).

We observe that:

$$
\chi\left(x, \frac{m}{p^{n}}\right)=\chi\left(\frac{x}{p^{n}}, m\right)=w_{m}\left(\frac{x}{p^{n}}\right), \quad \forall x \in\left[0, p^{n}\right), m, n \in \mathbb{Z}^{+}
$$

and:

$$
\chi(x \oplus y, z)=\chi(x, z) \chi(y, z), \quad \chi(x \ominus y, z)=\chi(x, z) \overline{\chi(y, z)}
$$

where $x, y, z \in \mathbb{R}^{+}$and $x \oplus y$ is $p$-adic irrational. It is well known that systems $\{\chi(\alpha, .)\}_{\alpha=0}^{\infty}$ and $\{\chi(\cdot, \alpha)\}_{\alpha=0}^{\infty}$ are orthonormal bases in $L^{2}[0,1]$ (see Golubov et al. [19]).

The Walsh-Fourier transform of a function $f \in L^{1}\left(\mathbb{R}^{+}\right) \cap L^{2}\left(\mathbb{R}^{+}\right)$is defined by:

$$
\hat{f}(\xi)=\int_{\mathbb{R}^{+}} f(x) \overline{\chi(x, \xi)} d x
$$

where $\chi(x, \xi)$ is given by Equation $(2)$. The Walsh-Fourier operator $\mathcal{F}: L^{1}\left(\mathbb{R}^{+}\right) \cap L^{2}\left(\mathbb{R}^{+}\right) \rightarrow L^{2}\left(\mathbb{R}^{+}\right)$, $\mathcal{F} f=\hat{f}$, extends uniquely to the whole space $L^{2}\left(\mathbb{R}^{+}\right)$. The properties of the Walsh-Fourier transform are quite similar to those of the classic Fourier transform (see $[19,20])$. In particular, if $f \in L^{2}\left(\mathbb{R}^{+}\right)$, then $\hat{f} \in L^{2}\left(\mathbb{R}^{+}\right)$and:

$$
\|\hat{f}\|_{L^{2}\left(\mathbb{R}^{+}\right)}=\|f\|_{L^{2}\left(\mathbb{R}^{+}\right)}
$$

Moreover, if $f \in L^{2}[0,1]$, then we can define the Walsh-Fourier coefficients of $f$ as:

$$
\hat{f}(n)=\int_{0}^{1} f(x) \overline{w_{n}(x)} d x
$$

The series $\sum_{n \in \mathbb{Z}^{+}} \hat{f}(n) w_{n}(x)$ is called the Walsh-Fourier series of $f$. Therefore, from the standard $L^{2}$-theory, we conclude that the Walsh-Fourier series of $f$ converges to $f$ in $L^{2}[0,1]$, and Parseval's identity holds:

$$
\|f\|_{2}^{2}=\int_{0}^{1}|f(x)|^{2} d x=\sum_{n \in \mathbb{Z}^{+}}|\hat{f}(n)|^{2}
$$

By $p$-adic interval $I \subset \mathbb{R}^{+}$of range $n$, we mean intervals of the form:

$$
I=I_{n}^{k}=\left[k p^{-n},(k+1) p^{-n}\right), \quad k \in \mathbb{Z}^{+}
$$

The $p$-adic topology is generated by the collection of $p$-adic intervals, and each $p$-adic interval is both open and closed under the $p$-adic topology (see [19]). The family $\left\{\left[0, p^{-j}\right): j \in \mathbb{Z}\right\}$ forms a fundamental system of the $p$-adic topology on $\mathbb{R}^{+}$. Therefore, the generalized Walsh functions $w_{j}(x), 0 \leq j \leq p^{n}-1$, assume constant values on each $p$-adic interval $I_{n}^{k}$ and, hence, continuous on these intervals. Thus, $w_{j}(x)=1$ for $x \in I_{n}^{0}$. 
Let $\mathcal{E}_{n}\left(\mathbb{R}^{+}\right)$be the space of $p$-adic entire functions of order $n$, that is the set of all functions that are constant on all $p$-adic intervals of range $n$. Thus, for every $f \in \mathcal{E}_{n}\left(\mathbb{R}^{+}\right)$, we have:

$$
f(x)=\sum_{k \in \mathbb{Z}^{+}} f\left(p^{-n} k\right) \chi_{I_{n}^{k}}(x), \quad x \in \mathbb{R}^{+}
$$

Clearly, each Walsh function of order up to $p^{n-1}$ belongs to $\mathcal{E}_{n}\left(\mathbb{R}^{+}\right)$. The set $\mathcal{E}\left(\mathbb{R}^{+}\right)$of $p$-adic entire functions on $\mathbb{R}^{+}$is the union of all of the spaces $\mathcal{E}_{n}\left(\mathbb{R}^{+}\right)$. It is clear that $\mathcal{E}\left(\mathbb{R}^{+}\right)$is dense in $L^{p}\left(\mathbb{R}^{+}\right), 1 \leq p<\infty$, and each function in $\mathcal{E}\left(\mathbb{R}^{+}\right)$is of compact support.

For $j \in \mathbb{N}_{0}$, let $\mathcal{N}_{j}$ denote a full collection of coset representatives of $\mathbb{Z}^{+} / p^{j} \mathbb{Z}^{+}$, i.e.,

$$
\mathcal{N}_{j}=\left\{0,1,2, \ldots, p^{j}-1\right\}, \quad j \geq 0
$$

Then, $\mathbb{Z}^{+}=\bigcup_{n \in \mathcal{N}_{j}}\left(n+p^{j} \mathbb{Z}^{+}\right)$, and for any distinct $n_{1}, n_{2} \in \mathcal{N}_{j}$, we have $\left(n_{1}+p^{j} \mathbb{Z}^{+}\right) \cap$ $\left(n_{2}+p^{j} \mathbb{Z}^{+}\right)=\emptyset$. Thus, every non-negative integer $k$ can uniquely be written as $k=r p^{j}+s$, where $r \in \mathbb{Z}^{+}, s \in \mathcal{N}_{j}$. Further, a bounded function $g: \mathbb{R}^{+} \rightarrow \mathbb{R}^{+}$is said to be a radially-decreasing $L^{1}$-majorant of $f(x) \in L^{2}\left(\mathbb{R}^{+}\right)$if $|f(x)| \leq g(x), g \in L^{1}\left(\mathbb{R}^{+}\right)$, and $g(0)<\infty$.

For $j \in \mathbb{Z}$ and $y \in \mathbb{R}^{+}$, we define the dilation $D_{j}$ and translation operators $T_{y}$ as follows:

$$
D_{j} f(x)=p^{j / 2} f\left(p^{j} x\right) \quad \text { and } \quad T_{y} f(x)=f(x \ominus y), \quad f \in L^{2}\left(\mathbb{R}^{+}\right)
$$

For given $\Psi:=\left\{\psi_{1}, \ldots, \psi_{L}\right\} \subset L^{2}\left(\mathbb{R}^{+}\right)$, define the wavelet system:

$$
\mathcal{F}(\Psi)=\left\{\psi_{\ell, j, k}(x):=p^{j / 2} \psi_{\ell}\left(p^{j} x \ominus k\right), j \in \mathbb{Z}, k \in \mathbb{Z}^{+}, \ell=1,2, \ldots, L\right\}
$$

The wavelet system $\mathcal{F}(\Psi)$ is called a wavelet frame, if there exist positive constants $A$ and $B$, such that:

$$
A\|f\|_{2}^{2} \leq \sum_{\ell=1}^{L} \sum_{j \in \mathbb{Z}} \sum_{k \in \mathbb{Z}^{+}}\left|\left\langle f, \psi_{\ell, j, k}\right\rangle\right|^{2} \leq B\|f\|_{2}^{2}
$$

holds for every $f \in L^{2}\left(\mathbb{R}^{+}\right)$, and we call the optimal constants $A$ and $B$ the lower frame bound and the upper frame bound, respectively. A tight wavelet frame refers to the case when $A=B$, and a Parseval wavelet frame refers to the case when $A=B=1$. On the other hand, if only the right-hand side of the above double inequality holds, then we say $\mathcal{F}(\Psi)$ is a Bessel sequence. If both $\mathcal{F}(\Psi)$ and $\mathcal{F}(\tilde{\Psi})$ are wavelet frames and for any $f \in L^{2}\left(\mathbb{R}^{+}\right)$, we have the reconstruction formula:

$$
f=\sum_{\ell=1}^{L} \sum_{j \in \mathbb{Z}} \sum_{k \in \mathbb{Z}^{+}}\left\langle f, \tilde{\psi}_{\ell, j, k}\right\rangle \psi_{\ell, j, k}
$$

in the $L^{2}$-sense; then, we say that $\mathcal{F}(\tilde{\Psi})$ is a dual wavelet frame of $\mathcal{F}(\Psi)$ (and vice versa), or we simply say that $(\mathcal{F}(\Psi), \mathcal{F}(\tilde{\Psi}))$ is a pair of dual framelets.

Wavelets and tight wavelet frames are often derived from refinable functions and wavelet masks. A compactly supported function $\phi(x) \in L^{2}\left(\mathbb{R}^{+}\right)$is called a $p$-refinable function, if it satisfies an equation of the type:

$$
\phi(x)=p \sum_{k=0}^{p^{n}-1} c_{k} \phi(p x \ominus k), \quad x \in \mathbb{R}^{+}
$$


where $c_{k}$ are complex coefficients. In the Fourier domain, the above refinement equation can be written as:

$$
\hat{\phi}(\xi)=h_{0}\left(\frac{\xi}{p}\right) \hat{\phi}\left(\frac{\xi}{p}\right)
$$

where:

$$
h_{0}(\xi)=\sum_{k=0}^{p^{n}-1} c_{k} \overline{w_{k}(\xi)}
$$

is a generalized Walsh polynomial, which is called the mask or symbol of the $p$-refinable function $\phi$ and is of course a $p$-adic step function. Observe that $w_{k}(0)=\hat{\phi}(0)=1$. By letting $\xi=0$ in Equations (11) and (12), we obtain $\sum_{k=0}^{p^{n}-1} c_{k}=1$. Since $\phi$ is compactly supported and in fact $\operatorname{supp} \phi \subset\left[0, p^{n-1}\right)$, therefore $\hat{\phi} \in \mathcal{E}_{n-1}\left(\mathbb{R}^{+}\right)$, and hence, as a result, $\hat{\phi}(\xi)=1$ for all $\xi \in\left[0, p^{1-n}\right)$ as $\hat{\phi}(0)=1$.

Suppose $\Psi=\left\{\psi_{1}, \ldots, \psi_{L}\right\}$ is a set of $p$-MRA functions derived from:

$$
\hat{\psi}_{\ell}(\xi)=h_{\ell}\left(\frac{\xi}{p}\right) \hat{\phi}\left(\frac{\xi}{p}\right)
$$

where:

$$
h_{\ell}(\xi)=\sum_{k=0}^{p^{n}-1} d_{k}^{\ell} \overline{w_{k}(\xi)}, \quad \ell=1, \ldots, L
$$

are the generalized Walsh polynomials, called the framelet symbols or wavelet masks. With $h_{\ell}(\xi), \ell=0,1, \ldots, L, L \geq p-1$ as the Walsh polynomials (wavelet masks), we formulate the matrix $\mathcal{M}(\xi)$ as:

$$
\mathcal{M}(\xi)=\left(\begin{array}{cccc}
h_{0}(\xi) & h_{0}(\xi \oplus 1 / p) & \ldots & h_{0}(\xi \oplus(p-1) / p) \\
h_{1}(\xi) & h_{1}(\xi \oplus 1 / p) & \ldots & h_{1}(\xi \oplus(p-1) / p) \\
\vdots & \vdots & \ddots & \vdots \\
h_{L}(\xi) & h_{L}(\xi \oplus 1 / p) & \ldots & h_{L}(\xi \oplus(p-1) / p)
\end{array}\right)
$$

The so-called unitary extension principle (UEP) provides a sufficient condition on $\Psi=\left\{\psi_{1}, \ldots, \psi_{L}\right\}$, such that the wavelet system $\mathcal{F}(\Psi)$ given by Equation (7) constitutes a tight frame for $L^{2}\left(\mathbb{R}^{+}\right)$. It is well known that in order to apply the UEP to derive a wavelet tight frame from a given refinable function, the corresponding refinement mask must satisfy:

$$
\sum_{k=0}^{p-1}\left|h_{0}(\xi \oplus k / p)\right|^{2} \leq 1, \quad \xi \in \mathbb{R}^{+}
$$

Recently, Shah [18] has given a general procedure for the construction of tight wavelet frames generated by the Walsh polynomials using unitary extension principles as:

Theorem 2.1: Let $\phi(x)$ be a compactly-supported refinable function, and $\hat{\phi}(0)=1$. Then, the wavelet system $\mathcal{F}(\Psi)$ given by (7) constitutes a Parseval frame in $L^{2}\left(\mathbb{R}^{+}\right)$provided the matrix $\mathcal{M}(\xi)$ as defined in Equation (15) satisfies:

$$
\mathcal{M}(\xi) \mathcal{M}^{*}(\xi)=I_{p}, \quad \text { for a.e. } \xi \in \sigma\left(V_{0}\right)
$$


where $\sigma\left(V_{0}\right):=\left\{\xi \in[0,1]: \sum_{k \in \mathbb{Z}^{+}}|\hat{\phi}(\xi \oplus k)|^{2} \neq 0\right\}$.

\section{Periodic Wavelet Frames Related to the Walsh Polynomials}

For any $f \in L^{1}\left(\mathbb{R}^{+}\right)$, we define the periodic version of $f$ as:

$$
f^{\text {per }}=\sum_{k \in \mathbb{Z}^{+}} f(x \oplus k)
$$

Then, it is easy to verify that $f^{\text {per }}$ is a well-defined locally-integrable function. With the same dilation and translation operators as in Equation (6), we define the periodic wavelet system as:

$$
\mathcal{F}\left(\Psi^{\mathrm{per}}\right):=\left\{\phi^{\mathrm{per}}, \psi_{\ell, j, k}^{\mathrm{per}}: 1 \leq \ell \leq L, j \in \mathbb{Z}^{+}, k \in \mathcal{N}_{j}\right\}
$$

First, we present an approach for constructing periodic wavelet frames generated by the Walsh polynomials on $\mathbb{R}^{+}$via the unitary extension principle (UEP). The following theorem is the main result of this section.

Theorem 3.1: Let $h_{0}, h_{1}, \ldots, h_{L}$ be the Walsh polynomials given by Equations (12) and (14), and let the wavelet system $\mathcal{F}(\Psi)$ given by Equation (7) form a Parseval wavelet frame generated by the compactly-supported $p$-refinable function $\phi$. If $\left\{\phi, \psi_{1}, \ldots, \psi_{L}\right\} \subset L^{1}\left(\mathbb{R}^{+}\right) \cap L^{2}\left(\mathbb{R}^{+}\right)$and $\phi, \psi_{1}, \psi_{2}, \ldots, \psi_{L}$ have a common radial decreasing $L^{1}$-majorant, then the periodic wavelet system $\mathcal{F}\left(\Psi^{\text {per }}\right)$ given by Equation (18) generates a Parseval wavelet frame for $L^{2}[0,1]$.

We split the proof of Theorem 3.1 into several lemmas.

Lemma 3.2: Suppose that the periodic wavelet system $\mathcal{F}\left(\Psi^{\text {per }}\right)$ is as in Theorem 3.1. Then, for any function $f \in \mathcal{E}[0,1)$ and given $\delta>0$, there exists a positive integer $J \in \mathbb{N}$, such that:

$$
(1-\delta)\|f\|_{2}^{2} \leq \sum_{k \in \mathcal{N}_{j}}\left|\left\langle f, \phi_{j, k}^{\text {per }}\right\rangle\right|^{2} \leq(1+\delta)\|f\|_{2}^{2}, \quad \text { for all } j \geq J
$$

Proof: Let $S$ denote the support of the Walsh-Fourier coefficients $\{\hat{f}(n)\}_{n \in \mathbb{Z}^{+}}$. Then, we have:

$$
f(x)=\sum_{n \in S} \hat{f}(n) \overline{w_{n}(x)}
$$

Let:

$$
\phi_{j, k}^{\mathrm{per}}(x)=\sum_{n \in \mathbb{Z}^{+}} \hat{\phi}_{j, k}^{\mathrm{per}}(n) \overline{w_{n}(x)}
$$

where the Walsh-Fourier coefficients of the above series are given by:

$$
\hat{\phi}_{j, k}^{\text {per }}(n)=p^{-j / 2} \hat{\phi}\left(p^{-j} n\right) \overline{w_{k}\left(p^{-j} n\right)}
$$

Applying Parseval's formula to the above Walsh-Fourier series, we obtain: 


$$
\begin{aligned}
\sum_{k \in \mathcal{N}_{j}}\left|\left\langle f, \phi_{j, k}^{\mathrm{per}}\right\rangle\right|^{2} & =\sum_{k \in \mathcal{N}_{j}}\left|\sum_{n \in S} \hat{f}(n) \overline{\hat{\phi}_{j, k}^{\mathrm{per}}(n)}\right|^{2} \\
& =\sum_{k \in \mathcal{N}_{j}}\left|\sum_{n \in S} \hat{f}(n) p^{-j / 2} \overline{\hat{\phi}\left(p^{-j} n\right)} w_{k}\left(p^{-j} n\right)\right|^{2} \\
& =\sum_{k \in \mathcal{N}_{j}}\left|\sum_{n \in S} d_{n}(\hat{f}, \hat{\phi}) w_{k}\left(p^{-j} n\right)\right|^{2}
\end{aligned}
$$

where $d_{n}(\hat{f}, \hat{\phi})=p^{-j / 2} \hat{f}(n) \overline{\hat{\phi}\left(p^{-j} n\right)}$. As $S$ is a finite set, there exists a positive number $N$, such that $S \subseteq D(N)=\left\{k \in \mathbb{Z}^{+}:|k| \leq N\right\}$. Hence, there exists $J_{1} \geq 0$, such that for all $j \geq J_{1}$, the elements of $D(N)$ lie in different cosets of $\mathbb{Z}^{+} / p^{j} \mathbb{Z}^{+}$(see [13]). Thus, the cardinality of $\mathcal{E}\left(\mathbb{R}^{+}\right) \bigcap\left(k+p^{j} \mathbb{Z}^{+}\right)$is at most one for each $j \geq J_{1}, k \in \mathcal{N}_{j}$. Consequently, we have:

$$
\begin{aligned}
\sum_{k \in \mathcal{N}_{j}}\left|\left\langle f, \phi_{j, k}^{\text {per }}\right\rangle\right|^{2} & =\sum_{k \in \mathcal{N}_{j}} \sum_{m \in S} d_{m}(\hat{f}, \hat{\phi}) w_{k}\left(p^{-j} m\right) \sum_{n \in S} \overline{d_{n}(\hat{f}, \hat{\phi}) w_{k}\left(p^{-j} n\right)} \\
& =\sum_{m \in S} \sum_{n \in S} d_{m}(\hat{f}, \hat{\phi}) \overline{d_{n}(\hat{f}, \hat{\phi})} \sum_{k \in \mathcal{N}_{j}} w_{k}\left(p^{-j}(m-n)\right) \\
& =p^{-j} \sum_{n \in S}\left|d_{n}(\hat{f}, \hat{\phi})\right|^{2} \\
& =\sum_{n \in S}\left|p^{-j / 2} \hat{f}(n) \overline{\hat{\phi}\left(p^{-j} n\right)}\right|^{2}
\end{aligned}
$$

Since $\hat{\phi}(0)=\lim _{\xi \rightarrow 0} \hat{\phi}(\xi)=1$, therefore there exists a non-negative integer $J_{2}$, such that:

$$
(1-\delta) \leq\left|\hat{\phi}\left(p^{-j} n\right)\right|^{2} \leq(1+\delta), \quad \text { for all } j \geq J_{2}
$$

Let $J=\max \left\{J_{1}, J_{2}\right\}$, then with this choice of $j \geq J$, we obtain:

$$
(1-\delta) \sum_{n \in S}|\hat{f}(n)|^{2} \leq \sum_{k \in \mathcal{N}_{j}}\left|\left\langle f, \phi_{j, k}^{\mathrm{per}}\right\rangle\right|^{2} \leq(1+\delta) \sum_{n \in S}|\hat{f}(n)|^{2}
$$

By using Equation (4), we have:

$$
(1-\delta)\|f\|_{2}^{2} \leq \sum_{k \in \mathcal{N}_{j}}\left|\left\langle f, \phi_{j, k}^{\mathrm{per}}\right\rangle\right|^{2} \leq(1+\delta)\|f\|_{2}^{2}
$$

This completes the proof of Lemma 3.2.

Lemma 3.3: Let $h_{0}(\xi)$ be the refinement mask of a compactly-supported refinable function $\phi$ of an MRA, and let $h_{\ell}(\xi), \ell=1,2, \ldots, L$ be the wavelet masks. Moreover, if the wavelet system $\mathcal{F}(\Psi)$ given by Equation (7) forms a normalized tight wavelet frame for $L^{2}\left(\mathbb{R}^{+}\right)$, then for any $f \in L^{2}\left(\mathbb{R}^{+}\right)$, we have: 


$$
\sum_{k \in \mathbb{Z}^{+}}\left|\left\langle f, \phi_{j+1, k}\right\rangle\right|^{2}=\sum_{k \in \mathbb{Z}^{+}}\left|\left\langle f, \phi_{j, k}\right\rangle\right|^{2}+\sum_{\ell=1}^{L} \sum_{k \in \mathbb{Z}^{+}}\left|\left\langle f, \psi_{\ell, j, k}\right\rangle\right|^{2}
$$

Proof: For any $f \in L^{2}\left(\mathbb{R}^{+}\right)$and $j \in \mathbb{Z}^{+}$, define the linear operators $P_{j}$ and $Q_{j}$ as:

$$
P_{j} f(x)=\sum_{k \in \mathbb{Z}^{+}}\left\langle f, \phi_{j, k}\right\rangle \phi_{j, k}(x), \quad Q_{j} f(x)=\sum_{\ell=1}^{L} \sum_{k \in \mathbb{Z}^{+}}\left\langle f, \psi_{\ell, j, k}\right\rangle \psi_{\ell, j, k}(x)
$$

Since $\mathcal{E}\left(\mathbb{R}^{+}\right)$is a dense subset of $L^{2}\left(\mathbb{R}^{+}\right)$, it is sufficient to prove that:

$$
\left\langle P_{j} f, f\right\rangle+\left\langle Q_{j} f, f\right\rangle=\left\langle P_{j+1} f, f\right\rangle
$$

holds for all of the functions $f$ in $\mathcal{E}\left(\mathbb{R}^{+}\right)$. Therefore, for all $f \in \mathcal{E}\left(\mathbb{R}^{+}\right)$and $j \in \mathbb{Z}, k \in \mathbb{Z}^{+}$, we obtain the following equality by using Parseval's formula:

$$
\begin{aligned}
\left\langle P_{j} f, f\right\rangle & =p^{j} \int_{0}^{1}\left|\sum_{m \in \mathbb{Z}^{+}} \hat{f}\left(p^{j}(\xi \oplus m)\right) \overline{\hat{\phi}(\xi \oplus m)}\right|^{2} d \xi \\
& =\int_{0}^{p^{j}}\left|\sum_{m \in \mathbb{Z}^{+}} \hat{f}\left(\xi \oplus p^{j} m\right) \overline{\hat{\phi}\left(p^{-j} \xi \oplus m\right)}\right|^{2} d \xi
\end{aligned}
$$

By taking advantage of the periodicity of the Walsh polynomial $h_{0}(\xi)$, we obtain:

$$
\begin{aligned}
& \left\langle P_{j} f, f\right\rangle=\int_{0}^{p^{j}}\left|\sum_{m \in \mathbb{Z}^{+}} \hat{f}\left(\xi \oplus p^{j} m\right) \overline{\hat{\phi}\left(p^{-j-1} \xi \oplus p^{-1} m\right)} \overline{h_{0}\left(p^{-j-1} \xi \oplus p^{-1} m\right)}\right|^{2} d \xi \\
& =\int_{0}^{p^{j}} \mid \sum_{m \in \mathbb{Z}^{+}} \sum_{n \in \mathcal{N}_{1}} \hat{f}\left(\xi \oplus p^{j}(p m \oplus n)\right) \overline{\hat{\phi}\left(p^{-j-1} \xi \oplus p^{-1}(p m \oplus n)\right)} \\
& \times\left.\overline{h_{0}\left(p^{-j-1} \xi \oplus p^{-1}(p m \oplus n)\right)}\right|^{2} d \xi \\
& =\int_{0}^{p^{j}} \mid \sum_{m \in \mathbb{Z}^{+}} \sum_{n \in \mathcal{N}_{1}} \hat{f}\left(\xi \oplus p^{j}(p m \oplus n)\right) \overline{\hat{\phi}\left(p^{-j-1} \xi \oplus p^{-1}(p m \oplus n)\right)} \\
& \times\left.\overline{h_{0}\left(p^{-j-1} \xi \oplus p^{-1} n\right)}\right|^{2} d \xi \\
& =\int_{0}^{p^{j}}\left|\sum_{n \in \mathcal{N}_{1}} R_{f, \phi}^{j}(n, \xi) \overline{h_{0}\left(p^{-j-1} \xi \oplus p^{-1} n\right)}\right|^{2} d \xi
\end{aligned}
$$

where:

$$
R_{f, \phi}^{j}(n, \xi)=\sum_{m \in \mathbb{Z}^{+}} \hat{f}\left(\xi \oplus p^{j}(p m \oplus n)\right) \overline{\hat{\phi}\left(p^{-j-1} \xi \oplus p^{-1}(p m \oplus n)\right)}
$$

Proceeding on similar lines as above, we can have:

$$
\left\langle Q_{j} f, f\right\rangle=\sum_{\ell=1}^{L} \int_{0}^{p^{j}}\left|\sum_{n \in \mathcal{N}_{1}} R_{f, \phi}^{j}(n, \xi) \overline{h_{\ell}\left(p^{-j-1} \xi \oplus p^{-1} n\right)}\right|^{2} d \xi
$$


Therefore, we have:

$$
\begin{aligned}
\left\langle P_{j} f, f\right\rangle+\left\langle Q_{j} f, f\right\rangle & \left.\int_{0}^{p^{j}}\left\{\sum_{n \in \mathcal{N}_{1}} R_{f, \phi}^{j}(n, \xi) \overline{h_{0}\left(p^{-j-1} \xi \oplus p^{-1} n\right)}\right\} \overline{\left\{\sum_{n^{\prime} \in \mathcal{N}_{1}} R_{f, \phi}^{j}\left(n^{\prime}, \xi\right) \overline{h_{0}\left(p^{-j-1} \xi \oplus p^{-1} n^{\prime}\right)}\right.}\right\} \\
& \left.+\sum_{\ell=1}^{L} \int_{0}^{p^{j}}\left\{\sum_{n \in \mathcal{N}_{1}} R_{f, \phi}^{j}(n, \xi) \overline{h_{\ell}\left(p^{-j-1} \xi \oplus p^{-1} n\right)}\right\} \overline{\left\{\sum_{n^{\prime} \in \mathcal{N}_{1}} R_{f, \phi}^{j}\left(n^{\prime}, \xi\right) \overline{h_{\ell}\left(p^{-j-1} \xi \oplus p^{-1} n^{\prime}\right)}\right.}\right\} \\
= & \int_{0}^{p^{j}}\left\{\sum_{n \in \mathcal{N}_{1}} \sum_{n^{\prime} \in \mathcal{N}_{1}} R_{f, \phi}^{j}(n, \xi) \overline{R_{f, \phi}^{j}\left(n^{\prime}, \xi\right)}\right\}\left\{\sum_{\ell=0}^{L} h_{\ell}\left(p^{-j-1} \xi \oplus p^{-1} n^{\prime}\right) \overline{h_{\ell}\left(p^{-j-1} \xi \oplus p^{-1} n\right)}\right\}
\end{aligned}
$$

Since the UEP condition Equation (17) is equivalent to:

$$
\sum_{\ell=0}^{L} h_{\ell}\left(p^{-j-1} \xi \oplus p^{-1} n^{\prime}\right) \overline{h_{\ell}\left(p^{-j-1} \xi \oplus p^{-1} n\right)}=\delta_{n, n^{\prime}}
$$

therefore, we have:

$$
\begin{aligned}
\left\langle P_{j} f, f\right\rangle+\left\langle Q_{j} f, f\right\rangle & =\int_{0}^{p^{j}} \sum_{n \in \mathcal{N}_{1}}\left|R_{f, \phi}^{j}(n, \xi)\right|^{2} d \xi \\
& =\int_{0}^{p^{j}} \sum_{n \in \mathcal{N}_{1}}\left|\sum_{m \in \mathbb{Z}^{+}} \hat{f}\left(\xi \oplus p^{j}(p m \oplus n)\right) \overline{\hat{\phi}\left(p^{-j-1} \xi \oplus p^{-1}(p m \oplus n)\right)}\right|^{2} d \xi \\
& =\sum_{n \in \mathcal{N}_{1}} \int_{p^{j} n}^{p^{j}(1+n)}\left|\sum_{m \in \mathbb{Z}^{+}} \hat{f}\left(\xi \oplus p^{j+1} m\right) \overline{\hat{\phi}\left(p^{-j-1} \xi \oplus m\right)}\right|^{2} d \xi \\
& =\int_{0}^{p^{j+1}}\left|\sum_{m \in \mathbb{Z}^{+}} \hat{f}\left(\xi \oplus p^{j+1} m\right) \overline{\hat{\phi}\left(p^{-j-1} \xi \oplus m\right)}\right|^{2} d \xi \\
& =\left\langle P_{j+1} f, f\right\rangle
\end{aligned}
$$

and hence, we get the desired result.

Lemma 3.4: Let $\phi \in L^{2}\left(\mathbb{R}^{+}\right)$be a compactly-supported refinable function with refinement mask $h_{0}(\xi)$, and let the wavelet system $\mathcal{F}(\Psi)$ given by Equation (7) constitute a Parseval wavelet frame for $L^{2}\left(\mathbb{R}^{+}\right)$. Moreover, if $\left\{\phi, \psi_{1}, \ldots, \psi_{L}\right\} \subset L^{1}\left(\mathbb{R}^{+}\right) \cap L^{2}\left(\mathbb{R}^{+}\right)$and $\phi, \psi_{1}, \psi_{2}, \ldots, \psi_{L}$ have a common radial decreasing $L^{1}$-majorant, then we have:

$$
\sum_{k \in \mathcal{N}_{j}}\left|\left\langle f, \phi_{j+1, k}^{\text {per }}\right\rangle\right|^{2}=\sum_{k \in \mathcal{N}_{j}}\left|\left\langle f, \phi_{j, k}^{\text {per }}\right\rangle\right|^{2}+\sum_{\ell=1}^{L} \sum_{k \in \mathcal{N}_{j}}\left|\left\langle f, \psi_{\ell, j, k}^{\text {per }}\right\rangle\right|^{2}
$$


Proof: For any $f \in \mathcal{E}\left(\mathbb{R}^{+}\right)$and $j \in \mathbb{Z}^{+}$, we have:

$$
\sum_{k \in \mathcal{N}_{j}}\left|\left\langle f, \phi_{j, k}^{\mathrm{per}}\right\rangle\right|^{2}=\sum_{k \in \mathcal{N}_{j}}\left|\left\langle f, \sum_{n \in \mathbb{Z}^{+}} \phi_{j, k}(x \oplus n)\right\rangle\right|^{2}=\sum_{k \in \mathcal{N}_{j}}\left|\sum_{n \in \mathbb{Z}^{+}}\left\langle f, \phi_{j, k}(x \oplus n)\right\rangle\right|^{2}
$$

The change of the summation and the integration above is reasonable. In fact, we have:

$$
\begin{aligned}
\sum_{n \in \mathbb{Z}^{+}} \int_{0}^{1}\left|f(x) \overline{\phi_{j, k}(x \oplus n)}\right| d x & \leq\|f\|_{L^{\infty}[0,1]} \int_{\mathbb{R}^{+}}\left|\phi_{j, k}(x)\right| d x \\
& =\|f\|_{L^{\infty}[0,1]} p^{-j / 2} \int_{\mathbb{R}^{+}}|\phi(x)| d x \\
& <\infty
\end{aligned}
$$

We can also deduce that the series:

$$
\sum_{k \in \mathcal{N}_{j}} \sum_{m \in \mathbb{Z}^{+}} \sum_{n \in \mathbb{Z}^{+}}\left\langle f, \phi_{j, k}(x \oplus m)\right\rangle \overline{\left\langle f, \phi_{j, k}(x \oplus n)\right\rangle}
$$

is absolutely convergent. Therefore, the series can be rearranged as follows:

$$
\begin{aligned}
\sum_{k \in \mathcal{N}_{j}}\left|\left\langle f, \phi_{j, k}^{\mathrm{per}}\right\rangle\right|^{2} & =\sum_{k \in \mathcal{N}_{j}} \sum_{m \in \mathbb{Z}^{+}} \sum_{n \in \mathbb{Z}^{+}}\left\langle f, \phi_{j, k}(x \oplus m)\right\rangle \overline{\left\langle f, \phi_{j, k}(x \oplus n)\right\rangle} \\
& =\sum_{k \in \mathcal{N}_{j}} \sum_{m \in \mathbb{Z}^{+}} \sum_{n \in \mathbb{Z}^{+}}\left\langle f, \phi_{j, k}(x \oplus m)\right\rangle \overline{\left\langle f, \phi_{j, k}(x \oplus m \oplus n)\right\rangle}
\end{aligned}
$$

For $n \in \mathbb{Z}^{+}$, we define:

$$
F_{n}(x)=f(x) \chi_{[0,1]+n}(x)
$$

where $\chi(x)$ is the characteristic function. Using the fact that $\phi_{j, k}(x \oplus n)=\phi_{j, k-p^{j} n}(x)$, we have:

$$
\begin{aligned}
\sum_{k \in \mathcal{N}_{j}}\left|\left\langle f, \phi_{j, k}^{\mathrm{per}}\right\rangle\right|^{2} & =\sum_{k \in \mathcal{N}_{j}} \sum_{m \in \mathbb{Z}^{+}} \sum_{n \in \mathbb{Z}^{+}}\left\{\int_{0}^{1} f(x) \overline{\phi_{j, k}(x \oplus m)} d x\right\} \overline{\left\{\int_{0}^{1} f(x) \overline{\phi_{j, k}(x \oplus m \oplus n)} d x\right\}} \\
& =\sum_{k \in \mathcal{N}_{j}} \sum_{m \in \mathbb{Z}^{+}} \sum_{n \in \mathbb{Z}^{+}}\left\{\int_{0}^{1} f\left(x \overline{\phi_{j, k}(x \oplus m)} d x\right\} \overline{\left\{\int_{0}^{1+n} f(x) \overline{\phi_{j, k}(x \oplus m)} d x\right\}}\right. \\
= & \sum_{k \in \mathcal{N}_{j}} \sum_{m \in \mathbb{Z}^{+}} \sum_{n \in \mathbb{Z}^{+}}\left\{\int_{\mathbb{R}^{+}} F_{0}(x) \overline{\phi_{j, k}(x \oplus m)} d x\right\} \overline{\left\{\int_{\mathbb{R}^{+}} F_{n}(x) \overline{\phi_{j, k}(x \oplus m)} d x\right\}} \\
= & \sum_{k \in \mathcal{N}_{j}} \sum_{m \in \mathbb{Z}^{+}} \sum_{n \in \mathbb{Z}^{+}}\left\langle F_{0}, \phi_{j, k-p^{j} m}\right\rangle \overline{\left\langle F_{n}, \phi_{j, k-p^{j} m}\right\rangle} \\
= & \sum_{k \in \mathbb{Z}^{+}} \sum_{n \in \mathbb{Z}^{+}}\left\langle F_{0}, \phi_{j, k}\right\rangle \overline{\left\langle F_{n}, \phi_{j, k}\right\rangle}
\end{aligned}
$$

Similarly, for each $\ell=1,2, \ldots, L$, we have: 


$$
\sum_{k \in \mathcal{N}_{j}}\left|\left\langle f, \psi_{\ell, j, k}^{\mathrm{per}}\right\rangle\right|^{2}=\sum_{k \in \mathbb{Z}^{+}} \sum_{n \in \mathbb{Z}^{+}}\left\langle F_{0}, \psi_{\ell, j, k}\right\rangle \overline{\left\langle F_{n}, \psi_{\ell, j, k}\right\rangle}
$$

The application of Lemma 3.3 yields:

$$
\begin{aligned}
\sum_{k \in \mathcal{N}_{j}}\left|\left\langle f, \phi_{j, k}^{\mathrm{per}}\right\rangle\right|^{2} & +\sum_{\ell=1}^{L} \sum_{k \in \mathcal{N}_{j}}\left|\left\langle f, \psi_{\ell, j, k}^{\mathrm{per}}\right\rangle\right|^{2} \\
& =\sum_{k \in \mathbb{Z}^{+}} \sum_{n \in \mathbb{Z}^{+}}\left\langle F_{0}, \phi_{j, k}\right\rangle \overline{\left\langle F_{n}, \phi_{j, k}\right\rangle}+\sum_{\ell=1}^{L} \sum_{k \in \mathbb{Z}^{+}} \sum_{n \in \mathbb{Z}^{+}}\left\langle F_{0}, \psi_{\ell, j, k}\right\rangle \overline{\left\langle F_{n}, \psi_{\ell, j, k}\right\rangle} \\
& =\sum_{k \in \mathbb{Z}^{+}} \sum_{n \in \mathbb{Z}^{+}}\left\langle F_{0}, \phi_{j+1, k}\right\rangle \overline{\left\langle F_{n}, \phi_{j+1, k}\right\rangle} \\
& =\sum_{k \in \mathcal{N}_{j}}\left|\left\langle f, \phi_{j+1, k}^{\mathrm{per}}\right\rangle\right|^{2}
\end{aligned}
$$

This completes the proof.

Proof of Theorem 3.1: For any function $f \in \mathcal{E}[0,1)$ and $\delta>0$, we can choose $J>0$ by Lemma 3.2, such that for all $j>J$, we have:

$$
(1-\delta)\|f\|_{2}^{2} \leq \sum_{k \in \mathcal{N}_{j}}\left|\left\langle f, \phi_{j, k}^{\mathrm{per}}\right\rangle\right|^{2} \leq(1+\delta)\|f\|_{2}^{2}
$$

For any $j \in \mathbb{Z}$, Lemma 3.4 implies that:

$$
\sum_{k \in \mathcal{N}_{j}}\left|\left\langle f, \phi_{j, k}^{\mathrm{per}}\right\rangle\right|^{2}=\sum_{k \in \mathcal{N}_{j-1}}\left|\left\langle f, \phi_{j-1, k}^{\mathrm{per}}\right\rangle\right|^{2}+\sum_{\ell=1}^{L} \sum_{k \in \mathcal{N}_{j-1}}\left|\left\langle f, \psi_{\ell, j-1, k}^{\mathrm{per}}\right\rangle\right|^{2}
$$

By repeating this argument on $\sum_{k \in \mathcal{N}_{j-1}}\left|\left\langle f, \phi_{j-1, k}^{\text {per }}\right\rangle\right|^{2}$, we obtain:

$$
\sum_{k \in \mathcal{N}_{j}}\left|\left\langle f, \phi_{j, k}^{\mathrm{per}}\right\rangle\right|^{2}=\left|\left\langle f, \phi^{\mathrm{per}}\right\rangle\right|^{2}+\sum_{\ell=1}^{L} \sum_{m=0}^{j-1} \sum_{k \in \mathcal{N}_{m}}\left|\left\langle f, \psi_{\ell, m, k}^{\mathrm{per}}\right\rangle\right|^{2}
$$

Therefore, we have:

$$
(1-\delta)\|f\|_{2}^{2} \leq\left|\left\langle f, \phi^{\mathrm{per}}\right\rangle\right|^{2}+\sum_{\ell=1}^{L} \sum_{m=0}^{j-1} \sum_{k \in \mathcal{N}_{m}}\left|\left\langle f, \psi_{\ell, m, k}^{\mathrm{per}}\right\rangle\right|^{2} \leq(1+\delta)\|f\|_{2}^{2}
$$

Letting $j \rightarrow \infty$, we obtain:

$$
(1-\delta)\|f\|_{2}^{2} \leq\left|\left\langle f, \phi^{\text {per }}\right\rangle\right|^{2}+\sum_{\ell=1}^{L} \sum_{m=0}^{\infty} \sum_{k \in \mathcal{N}_{m}}\left|\left\langle f, \psi_{\ell, m, k}^{\mathrm{per}}\right\rangle\right|^{2} \leq(1+\delta)\|f\|_{2}^{2}
$$

Since $\delta>0$ was arbitrary. Therefore, it follows that: 


$$
\left|\left\langle f, \phi^{\text {per }}\right\rangle\right|^{2}+\sum_{\ell=1}^{L} \sum_{m=0}^{\infty} \sum_{k \in \mathcal{N}_{m}}\left|\left\langle f, \psi_{\ell, m, k}^{\mathrm{per}}\right\rangle\right|^{2}=\|f\|_{2}^{2}
$$

This completes the proof of the Theorem 3.1.

\section{Dual Periodic Wavelet Frames Related to the Walsh Polynomials}

In this section, we construct dual periodic wavelet frames generated by the Walsh polynomials on $\mathbb{R}^{+}$ using the mixed extension principle (MEP). The following theorem is the main result of this section.

Theorem 4.1: Suppose that $\phi$ and $\tilde{\phi}$ are two compactly-supported refinable functions, and $h_{\ell}(\xi), \tilde{h}_{\ell}(\xi), \ell=0,1, \ldots, L$ are the Walsh polynomials. Let $\mathcal{F}(\Psi)$ and $\mathcal{F}(\tilde{\Psi})$ be a pair of dual wavelet frames for $L^{2}\left(\mathbb{R}^{+}\right)$generated by the mixed extension principle. Then, $\mathcal{F}\left(\Psi^{\text {per }}\right)$ and $\mathcal{F}\left(\tilde{\Psi}^{\text {per }}\right)$ form a pair of dual wavelet frames for $L^{2}[0,1]$.

We need the following lemmas, which are important for the proof of the main result.

Lemma 4.2: The sequences $\mathcal{F}\left(\Psi^{\text {per }}\right)$ and $\mathcal{F}\left(\tilde{\Psi}^{\text {per }}\right)$ are both Bessel sequences for $L^{2}[0,1]$.

Proof. To simplify expressions in the proof, we let:

$$
\begin{aligned}
P_{\Psi}(f) & =\sum_{\ell=1}^{L} \sum_{j \in \mathbb{Z}^{+}} \sum_{k \in \mathcal{N}_{j}}\left|\left\langle f, \psi_{\ell, j, k}^{\mathrm{per}}\right\rangle\right|^{2} \\
P_{\tilde{\Psi}}(f) & =\sum_{\ell=1}^{L} \sum_{j \in \mathbb{Z}^{+}} \sum_{k \in \mathcal{N}_{j}}\left|\left\langle f, \tilde{\psi}_{\ell, j, k}^{\mathrm{per}}\right\rangle\right|^{2}
\end{aligned}
$$

In order to prove that the sequences $\mathcal{F}\left(\Psi^{\text {per }}\right)$ and $\mathcal{F}\left(\tilde{\Psi}^{\text {per }}\right)$ are both Bessel sequences, we need to find out two positive numbers $C, \tilde{C}$, such that for any function $f \in \mathcal{E}\left(\mathbb{R}^{+}\right)$, we have:

$$
\begin{aligned}
& P_{\Psi}(f)+\left|\left\langle f, \phi^{\text {per }}\right\rangle\right|^{2} \leq C\|f\|_{2}^{2} \\
& P_{\tilde{\Psi}}(f)+\left|\left\langle f, \tilde{\phi}^{\text {per }}\right\rangle\right|^{2} \leq \tilde{C}\|f\|_{2}^{2}
\end{aligned}
$$

For any $f \in L^{2}[0,1]$, by the Parseval identity of the Walsh-Fourier series, we deduce that:

$$
\begin{aligned}
\left|\left\langle f, \phi^{\text {per }}\right\rangle\right|^{2} & =\left|\sum_{n \in \mathbb{Z}^{+}} \hat{f}(n) \overline{\hat{\phi}^{\text {per }}(n)}\right|^{2} \\
& =\left|\sum_{n \in \mathbb{Z}^{+}} \hat{f}(n) \hat{\phi}(n) w_{0}(n)\right|^{2} \\
& \leq \sum_{n \in \mathbb{Z}^{+}}|\hat{f}(n)|^{2} \sum_{n \in \mathbb{Z}^{+}}|\hat{\phi}(n)|^{2}
\end{aligned}
$$

Since $\lim _{n \rightarrow 0} \hat{\phi}(n)=1, n \in \mathbb{Z}^{+}$, so $\hat{\phi}$ is bounded on $\mathbb{Z}^{+}$; therefore, there exists $C_{1}>0$, such that $\sum_{n \in \mathbb{Z}^{+}}|\hat{\phi}(n)|^{2} \leq C_{1}$. Using Equation (4) and this estimate, Equation (27) reduces to:

$$
\left|\left\langle f, \phi^{\text {per }}\right\rangle\right|^{2} \leq C_{1}\|f\|_{2}^{2}
$$


Next, we compute $P_{\Psi}(f)$. Using the periodic property of the functions $\psi_{\ell, j, k}^{\text {per }}$, that is $\psi_{\ell, j, k}(x \oplus n)=\psi_{\ell, j, k-p^{j} n}(x), n \in \mathbb{Z}^{+}$, we have:

$$
\begin{aligned}
P_{\Psi}(f) & =\sum_{\ell=1}^{L} \sum_{j \in \mathbb{Z}^{+}} \sum_{k \in \mathcal{N}_{j}}\left(\sum_{n \in \mathbb{Z}^{+}}\left|\left\langle f, \psi_{\ell, j, k}(x \oplus n)\right\rangle\right|\right)^{2} \\
& =\sum_{\ell=1}^{L} \sum_{j \in \mathbb{Z}^{+}} \sum_{k \in \mathcal{N}_{j}}\left(\sum_{n \in \mathbb{Z}^{+}}\left|\int_{0}^{1} f(x) \overline{\psi_{\ell, j, k}(x \oplus n)} d x\right|\right)^{2} \\
& \leq C_{2} \sum_{\ell=1}^{L} \sum_{j \in \mathbb{Z}^{+}} \sum_{k \in \mathcal{N}_{j}} \sum_{n \in \mathbb{Z}^{+}}\left|\int_{0}^{1} f(x) \overline{\psi_{\ell, j, k}(x \oplus n)} d x\right|^{2} \\
& \leq C_{2} \sum_{\ell=1}^{L} \sum_{j \in \mathbb{Z}^{+}} \sum_{k \in \mathcal{N}_{j}}\left|\int_{0}^{1} f(x) \overline{\psi_{\ell, j, k}(x)} d x\right|^{2} \\
& =C_{2} \sum_{\ell=1}^{L} \sum_{j \in \mathbb{Z}^{+}} \sum_{k \in \mathcal{N}_{j}}\left|\int_{\mathbb{R}^{+}} f \cdot \chi_{[0,1]}(x) \overline{\psi_{\ell, j, k}(x)} d x\right|^{2}
\end{aligned}
$$

Since the wavelet system $\mathcal{F}(\Psi)$ is a Bessel sequence for $L^{2}\left(\mathbb{R}^{+}\right)$, thus we can deduce that there exists a positive number $C_{3}$, such that:

$$
P_{\Psi}(f) \leq C_{3}\left\|f \cdot \chi_{[0,1]}\right\|_{2}^{2}=C_{3}\|f\|_{2}^{2}
$$

Combining Equations (28) and (29), we get:

$$
P_{\Psi}(f)+\left|\left\langle f, \phi^{\mathrm{per}}\right\rangle\right|^{2} \leq\left(C_{1}+C_{3}\right)\|f\|_{2}^{2}=C_{4}\|f\|_{2}^{2}
$$

Similarly, we have:

$$
P_{\tilde{\Psi}}(f)+\left|\left\langle f, \tilde{\phi}^{\text {per }}\right\rangle\right|^{2} \leq \tilde{C}_{4}\|f\|_{2}^{2}
$$

This completes the proof of Lemma 4.2.

Lemma 4.3: If $f, g \in \mathcal{E}[0,1)$, i.e.,

$$
f(x)=\sum_{n \in \mathbb{Z}^{+}} \hat{f}(n) \overline{w_{n}(x)} \text { and } g(x)=\sum_{n \in \mathbb{Z}^{+}} \hat{g}(n) \overline{w_{n}(x)},
$$

where the sequences $\{\hat{f}(n)\}$ and $\{\hat{g}(n)\}$ have only finitely many non-zero terms. Then, the following formula holds:

$$
\langle f, g\rangle=\left\langle f, \phi_{j, k}^{\mathrm{per}}\right\rangle\left\langle\tilde{\phi}_{j, k}^{\mathrm{per}}, g\right\rangle+\sum_{\ell=1}^{L} \sum_{j \in \mathbb{Z}^{+}} \sum_{k \in \mathcal{N}_{j}}\left\langle f, \psi_{\ell, j, k}^{\mathrm{per}}\right\rangle\left\langle\tilde{\psi}_{\ell, j, k}^{\mathrm{per}}, g\right\rangle
$$

Proof: We split the proof of this results into three steps.

Step 1: We rearrange and rewrite the following series:

$$
\sum_{k \in \mathcal{N}_{j}}\left\langle f, \phi_{j, k}^{\mathrm{per}}\right\rangle\left\langle\tilde{\phi}_{j, k}^{\mathrm{per}}, g\right\rangle, \quad \sum_{k \in \mathcal{N}_{j}}\left\langle f, \psi_{\ell, j, k}^{\mathrm{per}}\right\rangle\left\langle\tilde{\psi}_{\ell, j, k}^{\mathrm{per}}, g\right\rangle
$$


Since both the functions $\phi$ and $\tilde{\phi}$ are compactly supported, thus it is possible to make a rearrangement in the above series:

$$
\begin{aligned}
\left\langle f, \phi_{j, k}^{\mathrm{per}}\right\rangle\left\langle\tilde{\phi}_{j, k}^{\mathrm{per}}, g\right\rangle & =\left(\sum_{m \in \mathbb{Z}^{+}}\left\langle f, \phi_{j, k}(x \oplus m)\right\rangle\right)\left(\sum_{n \in \mathbb{Z}^{+}}\left\langle\tilde{\phi}_{j, k}(x \oplus n), g\right\rangle\right) \\
& =\sum_{m \in \mathbb{Z}^{+}} \sum_{n \in \mathbb{Z}^{+}}\left\langle f, \phi_{j, k}(x \oplus m)\right\rangle\left\langle\tilde{\phi}_{j, k}(x \oplus n), g\right\rangle \\
& =\sum_{m \in \mathbb{Z}^{+}} \sum_{n \in \mathbb{Z}^{+}}\left\langle f, \phi_{j, k}(x \oplus m)\right\rangle\left\langle\tilde{\phi}_{j, k}(x \oplus m \oplus n), g\right\rangle
\end{aligned}
$$

For any $n \in \mathbb{Z}^{+}$, we define:

$$
F_{n}(x)=f(x) \chi_{[0,1]+n}(x), \quad G_{n}(x)=g(x) \chi_{[0,1]+n}(x)
$$

Since $f$ and $g$ are periodic functions, by Equations (31) and (32), we have:

$$
\begin{aligned}
\left\langle f, \phi_{j, k}^{\mathrm{per}}\right\rangle\left\langle\tilde{\phi}_{j, k}^{\mathrm{per}}, g\right\rangle & =\sum_{m \in \mathbb{Z}^{+}} \sum_{n \in \mathbb{Z}^{+}}\left(\int_{0}^{1} f(x) \overline{\phi_{j, k}(x \oplus m)} d x\right)\left(\int_{0}^{1} \tilde{\phi}_{j, k}(x \oplus m \oplus n) \overline{g(x)} d x\right) \\
& =\sum_{m \in \mathbb{Z}^{+}} \sum_{n \in \mathbb{Z}^{+}}\left(\int_{0}^{1} f(x) \overline{\phi_{j, k}(x \oplus m)} d x\right)\left(\int_{0}^{1+n} \tilde{\phi}_{j, k}(x \oplus m) \overline{g(x)} d x\right) \\
& =\sum_{m \in \mathbb{Z}^{+}} \sum_{n \in \mathbb{Z}^{+}}\left(\int_{\mathbb{R}^{+}} F_{0}(x) \overline{\phi_{j, k}(x \oplus m)} d x\right)\left(\int_{\mathbb{R}^{+}} \tilde{\phi}_{j, k}(x \oplus m) \overline{G_{n}(x)} d x\right)
\end{aligned}
$$

By summing Equation (33) over the set $\mathcal{N}_{j}$ and noting that $\phi_{j, k}(x \oplus m)=\phi_{j, k-p^{j} m}(x)$, we have:

$$
\begin{aligned}
\sum_{k \in \mathcal{N}_{j}}\left\langle f, \phi_{j, k}^{\text {per }}\right\rangle\left\langle\tilde{\phi}_{j, k}^{\text {per }}, g\right\rangle & =\sum_{k \in \mathcal{N}_{j}} \sum_{m \in \mathbb{Z}^{+}} \sum_{n \in \mathbb{Z}^{+}}\left\langle F_{0}, \phi_{j, k-p^{j} m}\right\rangle\left\langle\tilde{\phi}_{j, k-p^{j} m}, G_{n}\right\rangle \\
& =\sum_{n \in \mathbb{Z}^{+}} \sum_{k \in \mathbb{Z}^{+}}\left\langle F_{0}, \phi_{j, k}\right\rangle\left\langle\tilde{\phi}_{j, k}, G_{n}\right\rangle
\end{aligned}
$$

Similarly, for each $\ell=1,2, \ldots, L$, we have:

$$
\sum_{k \in \mathcal{N}_{j}}\left\langle f, \psi_{\ell, j, k}^{\mathrm{per}}\right\rangle\left\langle\tilde{\psi}_{\ell, j, k}^{\mathrm{per}}, g\right\rangle=\sum_{n \in \mathbb{Z}^{+}} \sum_{k \in \mathbb{Z}^{+}}\left\langle F_{0}, \psi_{\ell, j, k}\right\rangle\left\langle\tilde{\psi}_{\ell, j, k}, G_{n}\right\rangle
$$

Step 2: For any $J \geq 0$, we claim that:

$$
\left\langle f, \phi_{j, k}^{\mathrm{per}}\right\rangle\left\langle\tilde{\phi}_{j, k}^{\mathrm{per}}, g\right\rangle+\sum_{\ell=1}^{L} \sum_{j=0}^{J} \sum_{k \in \mathcal{N}_{j}}\left\langle f, \psi_{\ell, j, k}^{\mathrm{per}}\right\rangle\left\langle\tilde{\psi}_{\ell, j, k}^{\mathrm{per}}, g\right\rangle=\sum_{k \in \mathcal{N}_{J+1}}\left\langle f, \phi_{J+1, k}^{\mathrm{per}}\right\rangle\left\langle\tilde{\phi}_{J+1, k}^{\mathrm{per}}, g\right\rangle
$$

Taking the sum on the R.H.S of Equation (35) over $\ell=0,1, \ldots, L$, we have:

$$
Q_{j}=\sum_{\ell=0}^{L} \sum_{k \in \mathbb{Z}^{+}} \sum_{n \in \mathbb{Z}^{+}}\left\langle F_{0}, \psi_{\ell, j, k}\right\rangle\left\langle\tilde{\psi}_{\ell, j, k}, G_{n}\right\rangle
$$


By the Parseval identity of the Walsh-Fourier transform and Equation (13), we deduce that:

$$
\begin{aligned}
\left\langle F_{0}, \psi_{\ell, j, k}\right\rangle & =\left\langle\hat{F}_{0}, \hat{\psi}_{\ell, j, k}\right\rangle \\
& =p^{-j / 2} \int_{\mathbb{R}^{+}} \hat{F}_{0}(\xi) \overline{\hat{\psi}_{\ell}\left(p^{-j} \xi\right)} w_{k}\left(p^{-j} \xi\right) d \xi \\
& =p^{-j / 2} \int_{\mathbb{R}^{+}} \hat{F}_{0}(\xi) \overline{h_{\ell}\left(p^{-j-1} \xi\right)} \overline{\hat{\phi}\left(p^{-j-1} \xi\right)} w_{k}\left(p^{-j} \xi\right) d \xi \\
& =p^{-j / 2} \int_{0}^{p^{j}} \sum_{m \in \mathbb{Z}^{+}} \hat{F}_{0}\left(\xi \oplus p^{j} m\right) \overline{h_{\ell}\left(p^{-j-1} \xi \oplus p^{-1} m\right)} \\
& \times \overline{\hat{\phi}\left(p^{-j-1} \xi \oplus p^{-1} m\right)} w_{k}\left(p^{-j} \xi\right) d \xi
\end{aligned}
$$

Since $h_{\ell}(\xi), 1 \leq \ell \leq L$ are the Walsh polynomials (wavelet masks) associated with given wavelets $\psi_{\ell}$ and we know each $h_{\ell}(\xi)$ is bounded periodic on [0,1], therefore we have:

$$
\begin{gathered}
\int_{0}^{p^{j}}\left\{\sum_{m \in \mathbb{Z}^{+}}\left|\hat{F}_{0}\left(\xi \oplus p^{j} m\right) \overline{h_{\ell}\left(p^{-j-1} \xi \oplus p^{-1} m\right)} \overline{\hat{\phi}\left(p^{-j-1} \xi \oplus p^{-1} m\right)} w_{k}\left(p^{-j} \xi\right)\right|\right\} d \xi \\
\quad \leq\left\|h_{\ell}\right\|_{\infty} \int_{\mathbb{R}^{+}}\left|\hat{F}_{0}(\xi) \overline{\hat{\phi}\left(p^{-j-1} \xi\right)}\right| d \xi<\infty
\end{gathered}
$$

Therefore, the exchange of the integral and the summation is reasonable in the above formula. Again, by the periodicity of wavelet masks $h_{\ell}$, we infer that:

$$
\begin{aligned}
\left\langle F_{0}, \psi_{\ell, j, k}\right\rangle=p^{-j / 2} \int_{0}^{p^{j}} \sum_{m^{\prime} \in \mathbb{Z}^{+}} & \sum_{t \in \mathcal{N}_{1}} \hat{F}_{0}\left(\xi \oplus p^{j}\left(p m^{\prime} \oplus t\right)\right) w_{k}\left(p^{-j} \xi\right) \\
& \times \overline{h_{\ell}\left(p^{-j-1} \xi \oplus p^{-1}\left(p m^{\prime} \oplus t\right)\right)} \overline{\hat{\phi}\left(p^{-j-1} \xi \oplus p^{-1}\left(p m^{\prime} \oplus t\right)\right)} d \xi \\
=p^{-j / 2} \int_{0}^{p^{j}} \sum_{m^{\prime} \in \mathbb{Z}^{+}} & \sum_{t \in \mathcal{N}_{1}} \hat{F}_{0}\left(\xi \oplus p^{j}\left(p m^{\prime} \oplus t\right) w_{k}\left(p^{-j} \xi\right)\right. \\
& \times \overline{\hat{\phi}\left(p^{-j-1} \xi \oplus p^{-1}\left(p m^{\prime} \oplus t\right)\right.} \overline{h_{\ell}\left(p^{-j-1} \xi \oplus p^{-1} t\right)} d \xi
\end{aligned}
$$

Similarly, we have:

$$
\begin{aligned}
\left\langle\tilde{\psi}_{\ell, j, k}, G_{n}\right\rangle=p^{-j / 2} & \int_{0}^{p^{j}} \sum_{n^{\prime} \in \mathbb{Z}^{+}} \sum_{t^{\prime} \in \mathcal{N}_{1}} \hat{G}_{n}\left(\xi \oplus p^{j}\left(p n^{\prime} \oplus t^{\prime}\right)\right) w_{k}\left(p^{-j} \xi\right) \\
& \times \frac{\hat{\tilde{\phi}}\left(p^{-j-1} \xi \oplus p^{-1}\left(p n^{\prime} \oplus t^{\prime}\right)\right)}{\tilde{h}_{\ell}\left(p^{-j-1} \xi \oplus p^{-1} t^{\prime}\right)} d \xi
\end{aligned}
$$

Since $\left\{p^{-j / 2} w_{k}\left(p^{-j} \xi\right): k \in \mathbb{Z}^{+}\right\}$is an orthonormal basis for $L^{2}\left(p^{j}[0,1]\right)$, therefore, by Equations (38) and (39), together with the Parseval identity of the Walsh-Fourier series, we have:

$$
\begin{aligned}
\sum_{k \in \mathbb{Z}^{+}}\langle & \left.F_{0}, \psi_{\ell, j, k}\right\rangle\left\langle\tilde{\psi}_{\ell, j, k}, G_{n}\right\rangle \\
= & \int_{0}^{p^{j}}\left\{\sum_{m^{\prime} \in \mathbb{Z}^{+}} \sum_{t \in \mathcal{N}_{1}} \hat{F}_{0}\left(\xi \oplus p^{j}\left(p m^{\prime} \oplus t\right) \overline{h_{\ell}\left(p^{-j-1} \xi \oplus p^{-1} t\right)} \overline{\hat{\phi}\left(p^{-j-1} \xi \oplus p^{-1}\left(p m^{\prime} \oplus t\right)\right)}\right\}\right. \\
& \times\left\{\sum_{n^{\prime} \in \mathbb{Z}^{+}} \sum_{t^{\prime} \in \mathcal{N}_{1}} \overline{\hat{G}_{n}\left(\xi \oplus p^{j}\left(p n^{\prime} \oplus t^{\prime}\right)\right)} \tilde{h}_{\ell}\left(p^{-j-1} \xi \oplus p^{-1} t^{\prime}\right) \hat{\tilde{\phi}}\left(p^{-j-1} \xi \oplus p^{-1}\left(p n^{\prime} \oplus t^{\prime}\right)\right)\right\} d \xi
\end{aligned}
$$


Again, by Equation (36), we deduce that:

$$
\begin{array}{r}
Q_{j}=\sum_{n \in \mathbb{Z}^{+}} \int_{0}^{p^{j}} \sum_{m^{\prime} \in \mathbb{Z}^{+}} \sum_{n^{\prime} \in \mathbb{Z}^{+}} \sum_{t \in \mathcal{N}_{1}} \sum_{t^{\prime} \in \mathcal{N}_{1}} \hat{F}_{0}\left(\xi \oplus p^{j}\left(p m^{\prime} \oplus t\right) \overline{\hat{G}_{n}\left(\xi \oplus p^{j}\left(p n^{\prime} \oplus t^{\prime}\right)\right)}\right. \\
\times \overline{\hat{\phi}\left(p^{-j-1} \xi \oplus p^{-1}\left(p m^{\prime} \oplus t\right)\right)} \hat{\tilde{\phi}}\left(p^{-j-1} \xi \oplus p^{-1}\left(p n^{\prime} \oplus t^{\prime}\right)\right) \\
\times\left(\sum_{\ell=0}^{L} \overline{h_{\ell}\left(p^{-j-1} \xi \oplus p^{-1} t\right)} \tilde{h}_{\ell}\left(p^{-j-1} \xi \oplus p^{-1} t^{\prime}\right)\right) d \xi
\end{array}
$$

By the mixed extension principle (MEP) condition, we have:

$$
\sum_{\ell=0}^{L} \overline{h_{\ell}\left(p^{-j-1} \xi \oplus p^{-1} t\right)} \tilde{h}_{\ell}\left(p^{-j-1} \xi \oplus p^{-1} t^{\prime}\right)=\delta_{t, t^{\prime}}
$$

Therefore, we have:

$$
\begin{aligned}
Q_{j}=\sum_{n \in \mathbb{Z}^{+}} \int_{0}^{p^{j}} \sum_{m^{\prime} \in \mathbb{Z}^{+}} & \sum_{n^{\prime} \in \mathbb{Z}^{+}} \sum_{t \in \mathcal{N}_{1}} \hat{F}_{0}\left(\xi \oplus p^{j}\left(p m^{\prime} \oplus t\right)\right) \overline{\hat{G}_{n}\left(\xi \oplus p^{j}\left(p n^{\prime} \oplus t\right)\right)} \\
\times & \overline{\hat{\phi}\left(p^{-j-1} \xi \oplus p^{-1}\left(p m^{\prime} \oplus t\right)\right)} \hat{\tilde{\phi}}\left(p^{-j-1} \xi \oplus p^{-1}\left(p n^{\prime} \oplus t\right)\right) d \xi
\end{aligned}
$$

Letting:

$R_{j}(\xi)=\sum_{m^{\prime} \in \mathbb{Z}^{+}} \hat{F}_{0}\left(\xi \oplus p^{j+1} m^{\prime}\right) \overline{\hat{\phi}\left(p^{-j-1} \xi \oplus m^{\prime}\right)}, \quad S_{j, n}(\xi)=\sum_{n^{\prime} \in \mathbb{Z}^{+}} \overline{\hat{G}_{n}\left(\xi \oplus p^{j+1} n^{\prime}\right)} \hat{\tilde{\phi}}\left(p^{-j-1} \xi \oplus n^{\prime}\right)$

we conclude that:

$$
\begin{aligned}
Q_{j} & =\sum_{n \in \mathbb{Z}^{+}} \sum_{t \in \mathcal{N}_{1}} \int_{0}^{p^{j}} R_{j}\left(\xi \oplus p^{j} t\right) \overline{S_{j, n}\left(\xi \oplus p^{j} t\right)} d \xi \\
& =\sum_{n \in \mathbb{Z}^{+}} \sum_{t \in \mathcal{N}_{1}} \int_{p^{j}}^{(1+t) p^{j}} R_{j}(\xi) \overline{S_{j, n}(\xi)} d \xi \\
& =\sum_{n \in \mathbb{Z}^{+}} \int_{0}^{p^{j+1}} R_{j}(\xi) \overline{S_{j, n}(\xi)} d \xi
\end{aligned}
$$

Using the Parseval identity of the Walsh-Fourier series, we obtain:

$$
\begin{aligned}
& \int_{0}^{p^{j+1}} R_{j}(\xi) \overline{S_{j, n}(\xi)} d \xi \\
& =p^{-(j+1)} \sum_{k \in \mathbb{Z}^{+}} \int_{0}^{p^{j+1}} R_{j}(\xi) \overline{w_{p^{-(j+1)} k}(\xi)} d \xi \int_{0}^{p^{j+1}} \overline{S_{j, n}(\xi)} w_{p^{-(j+1)} k}(\xi) d \xi \\
& =p^{-(j+1)} \sum_{k \in \mathbb{Z}^{+}} \int_{\mathbb{R}^{+}} \hat{F}_{0}(\xi) \overline{\hat{\phi}\left(p^{-j-1} \xi\right)} \overline{w_{p^{-(j+1)}}(\xi)} d \xi \int_{\mathbb{R}^{+}} \overline{\hat{G}_{n}(\xi)} \hat{\tilde{\phi}}\left(p^{-j-1} \xi\right) w_{p^{-(j+1) k}}(\xi) d \xi
\end{aligned}
$$


Again, by the Parseval identity of the Walsh-Fourier transform, we have:

$$
Q_{j}=\sum_{k \in \mathbb{Z}^{+}} \sum_{n \in \mathbb{Z}^{+}}\left\langle F_{0}, \phi_{j+1, k}\right\rangle\left\langle\tilde{\phi}_{j+1, k}, G_{n}\right\rangle
$$

By Equations (36) and (40), it follows that:

$$
\begin{aligned}
& \sum_{k \in \mathbb{Z}^{+}} \sum_{n \in \mathbb{Z}^{+}}\left\langle F_{0}, \phi_{j+1, k}\right\rangle\left\langle\tilde{\phi}_{j+1, k}, G_{n}\right\rangle=\sum_{\ell=0}^{L} \sum_{k \in \mathbb{Z}^{+}} \sum_{n \in \mathbb{Z}^{+}}\left\langle F_{0}, \psi_{\ell, j, k}\right\rangle\left\langle\tilde{\psi}_{\ell, j, k}, G_{n}\right\rangle \\
& =\sum_{k \in \mathbb{Z}^{+}} \sum_{n \in \mathbb{Z}^{+}}\left\langle F_{0}, \phi_{j, k}\right\rangle\left\langle\tilde{\phi}_{j, k}, G_{n}\right\rangle+\sum_{\ell=1}^{L} \sum_{k \in \mathbb{Z}^{+}} \sum_{n \in \mathbb{Z}^{+}}\left\langle F_{0}, \psi_{\ell, j, k}\right\rangle\left\langle\tilde{\psi}_{\ell, j, k}, G_{n}\right\rangle
\end{aligned}
$$

Using Equations (34) and (35) in the above identity, we obtain:

$$
\sum_{k \in \mathcal{N}_{j+1}}\left\langle f, \phi_{j+1, k}^{\mathrm{per}}\right\rangle\left\langle\tilde{\phi}_{j+1, k}^{\mathrm{per}}, g\right\rangle=\sum_{k \in \mathcal{N}_{j}}\left\langle f, \phi_{j, k}^{\mathrm{per}}\right\rangle\left\langle\tilde{\phi}_{j, k}^{\mathrm{per}}, g\right\rangle+\sum_{\ell=1}^{L} \sum_{k \in \mathcal{N}_{j}}\left\langle f, \psi_{\ell, j, k}^{\mathrm{per}}\right\rangle\left\langle\tilde{\psi}_{\ell, j, k}^{\mathrm{per}}, g\right\rangle
$$

Since $\mathcal{N}_{j}=\{0\}$, when $j=0$, we have:

$$
\sum_{k \in \mathcal{N}_{1}}\left\langle f, \phi_{1, k}^{\mathrm{per}}\right\rangle\left\langle\tilde{\phi}_{1, k}^{\mathrm{per}}, g\right\rangle=\left\langle f, \phi_{0,0}^{\mathrm{per}}\right\rangle\left\langle\tilde{\phi}_{0,0}^{\mathrm{per}}, g\right\rangle+\sum_{\ell=1}^{L} \sum_{k \in \mathcal{N}_{1}}\left\langle f, \psi_{\ell, 0, k}^{\mathrm{per}}\right\rangle\left\langle\tilde{\psi}_{\ell, 0, k}^{\mathrm{per}}, g\right\rangle
$$

In general, for any $J \geq 0$, we have:

$$
\sum_{k \in \mathcal{N}_{J+1}}\left\langle f, \phi_{J+1, k}^{\mathrm{per}}\right\rangle\left\langle\tilde{\phi}_{J+1, k}^{\mathrm{per}}, g\right\rangle=\left\langle f, \phi_{j, k}^{\mathrm{per}}\right\rangle\left\langle\tilde{\phi}_{j, k}^{\mathrm{per}}, g\right\rangle+\sum_{\ell=1}^{L} \sum_{j=0}^{J} \sum_{k \in \mathcal{N}_{j}}\left\langle f, \psi_{\ell, j, k}^{\mathrm{per}}\right\rangle\left\langle\tilde{\psi}_{\ell, j, k}^{\mathrm{per}}, g\right\rangle
$$

Step 3: For $f, g \in \mathcal{E}[0,1)$, we have:

$$
T_{j}=\sum_{k \in \mathcal{N}_{j}}\left\langle f, \phi_{j, k}^{\mathrm{per}}\right\rangle\left\langle\tilde{\phi}_{j, k}^{\mathrm{per}}, g\right\rangle \rightarrow\langle f, g\rangle, \quad j \rightarrow \infty
$$

Since $f$ and $g$ are both periodic, hence there exists a non-negative integer $J$, such that:

$$
f(x)=\sum_{n \in \mathbb{Z}^{+}} \hat{f}(n) \overline{w_{n}(x)}, \quad g(x)=\sum_{n \in \mathbb{Z}^{+}} \hat{g}(n) \overline{w_{n}(x)}
$$

where $\hat{f}(n)=\hat{g}(n)=0, n \notin \mathcal{N}_{J}$. Again, let:

$$
\phi_{j, k}^{\mathrm{per}}(x)=\sum_{n \in \mathbb{Z}^{+}} \hat{\phi}_{j, k}^{\mathrm{per}}(n) \overline{w_{n}(x)}, \quad \tilde{\phi}_{j, k}^{\mathrm{per}}(x)=\sum_{n \in \mathbb{Z}^{+}} \hat{\tilde{\phi}}_{j, k}^{\mathrm{per}}(n) \overline{w_{n}(x)},
$$

where:

$$
\hat{\phi}_{j, k}^{\text {per }}(n)=p^{-j / 2} \hat{\phi}\left(p^{-j} n\right) \overline{w_{k}\left(p^{-j} n\right)}, \quad \hat{\tilde{\phi}}_{j, k}^{\text {per }}(n)=p^{-j / 2} \hat{\tilde{\phi}}\left(p^{-j} n\right) \overline{w_{k}\left(p^{-j} n\right)}
$$

Therefore, for $j \geq J$, we have:

$$
\left\langle f, \phi_{m, k}^{\mathrm{per}}\right\rangle=\sum_{n \in \mathcal{N}_{j}} \hat{f}(n) \overline{\hat{\phi}_{j, k}^{\mathrm{per}}(n)}=\sum_{n \in \mathcal{N}_{j}} \hat{f}(n) p^{-j / 2} \hat{\phi}\left(p^{-j} n\right) \overline{w_{k}\left(p^{-j} n\right)}
$$


Similarly, we have:

$$
\left\langle\tilde{\phi}_{m, k}^{\text {per }}, g\right\rangle=\sum_{n \in \mathcal{N}_{j}} \hat{g}(n) p^{-j / 2} \hat{\tilde{\phi}}\left(p^{-j} n\right) \overline{w_{k}\left(p^{-j} n\right)}, \quad j \geq J
$$

Hence, we conclude that for $j \geq J$, we have:

$$
\begin{aligned}
T_{j} & =\sum_{k \in \mathcal{N}_{j}}\left(\sum_{m \in \mathcal{N}_{j}} \hat{f}(m) p^{-j / 2} \hat{\phi}\left(p^{-j} m\right) \overline{w_{k}\left(p^{-j} m\right)}\right) \overline{\left(\sum_{n \in \mathcal{N}_{j}} \hat{g}(n) p^{-j / 2} \hat{\tilde{\phi}}\left(p^{-j} n\right) \overline{w_{k}\left(p^{-j} n\right)}\right)} \\
& =\sum_{m \in \mathcal{N}_{j}} \sum_{n \in \mathcal{N}_{j}} \hat{f}(m) \overline{\hat{g}(n)} \hat{\phi}\left(p^{-j} m\right) \overline{\hat{\tilde{\phi}}\left(p^{-j} n\right)}\left(\sum_{k \in \mathcal{N}_{j}} p^{-j} \overline{w_{k}\left(p^{-j}(m \ominus n)\right)}\right) \\
& =\sum_{n \in \mathcal{N}_{j}} \hat{f}(n) \overline{\hat{g}(n)} \hat{\phi}\left(p^{-j} n\right) \overline{\hat{\tilde{\phi}}\left(p^{-j} n\right)} \\
& =\sum_{n \in \mathcal{N}_{J}} \hat{f}(n) \overline{\hat{g}(n)} \hat{\phi}\left(p^{-j} n\right) \overline{\hat{\tilde{\phi}}\left(p^{-j} n\right)}
\end{aligned}
$$

Since $\lim _{\xi \rightarrow 0} \hat{\phi}(\xi)=\lim _{\xi \rightarrow 0} \hat{\tilde{\phi}}(\xi)=1$, we have:

$$
\lim _{j \rightarrow \infty} T_{j}=\sum_{n \in \mathcal{N}_{J}} \hat{f}(n) \overline{\hat{g}(n)}=\sum_{n \in \mathbb{Z}^{+}} \hat{f}(n) \overline{\hat{g}(n)}=\langle f, g\rangle
$$

From Equations (41) and (42), we deduce that:

$$
\left\langle f, \phi_{j, k}^{\mathrm{per}}\right\rangle\left\langle\tilde{\phi}_{j, k}^{\mathrm{per}}, g\right\rangle+\sum_{\ell=1}^{L} \sum_{j \in \mathbb{Z}^{+}} \sum_{k \in \mathcal{N}_{j}}\left\langle f, \psi_{\ell, j, k}^{\mathrm{per}}\right\rangle\left\langle\tilde{\psi}_{\ell, j, k}^{\mathrm{per}}, g\right\rangle=\langle f, g\rangle
$$

This completes the proof of the Lemma 4.3.

Proof of Theorem 4.1: By Lemma 4.2, it follows that the sequences $\mathcal{F}\left(\Psi^{\text {per }}\right)$ and $\mathcal{F}\left(\tilde{\Psi}^{\text {per }}\right)$ are both Bessel sequences for $L^{2}[0,1]$. By Lemma 4.3, we know that for any $f, g \in \mathcal{E}[0,1)$, Equation (30) holds. Again, since the set $\mathcal{E}[0,1)$ is dense in $L^{2}[0,1]$, it follows that the periodic wavelet systems $\mathcal{F}\left(\Psi^{\mathrm{per}}\right)$ and $\mathcal{F}\left(\tilde{\Psi}^{\text {per }}\right)$ constitute a pair of dual frames for $L^{2}[0,1]$. This completes the proof.

\section{Acknowledgments}

The authors express their gratitude to the anonymous referees for their careful reading and very useful comments, which improved the final version of this paper.

\section{Author Contributions}

There was equal contribution by the authors. 


\section{Conflicts of Interest}

The authors declare no conflict of interest.

\section{References}

1. Ron, A.; Shen, Z. Affine systems in $L^{2}\left(\mathbb{R}^{d}\right)$ : The analysis of the analysis operator. J. Funct. Anal. 1997, 148, 408-447.

2. Daubechies, I.; Han, B.; Ron, A.; Shen, Z. Framelets: MRA-based constructions of wavelet frames. Appl. Comput. Harmon. Anal. 2003, 14, 1-46.

3. Zhang, Z. Periodic wavelet frames. Adv. Comput. Math. 2005, 22, 165-180.

4. Zhang, Z.; Saito, N. Constructions of periodic wavelet frames using extension principles. Appl. Comput. Harmon. Anal. 2009, 27, 12-23.

5. Dong, B.; Ji, H.; Li, J.; Shen, Z.; Xu, Y. Wavelet frame based blind image inpainting. Appl. Comput. Harmon. Anal. 2012, 32, 268-279.

6. Farkov, Yu.A.; Lebedeva, E.A.; Skopina, M.A. Wavelet frames on Vilenkin groups and their approximation properties. Int. J. Wavelets Multiresolut. Inf. Process. 2015, 13, in press.

7. Goh, S.S.; Teo, K.M. Extension principles for tight wavelet frames of periodic functions. Appl. Comput. Harmon. Anal. 2008, 25, 168-186.

8. Li, Y.Z.; Jia, H.F. The construction of multivariate periodic wavelet bi-frames. J. Math. Anal. Appl. 2014, 412, 852-865.

9. Debnath, L.; Shah, F.A. Wavelet Transforms and Their Applications; Birkhäuser: New York, NY, USA, 2015.

10. Lang, W.C. Orthogonal wavelets on the Cantor dyadic group. SIAM J. Math. Anal. 1996, 27, $305-312$.

11. Daubechies, I. Ten Lectures on Wavelets; SIAM: Philadelphia, PA, USA, 1992.

12. Kozyrev, S.V. Wavelet analysis as a $p$-adic spectral analysis. Izv. Akad. Nauk, Ser. Mat. 2002, 66, 149-158.

13. Farkov, Yu.A. On wavelets related to Walsh series. J. Approx. Theory 2009, 161, 259-279.

14. Shah, F.A.; Debnath, L. Dyadic wavelet frames on a half-line using the Walsh-Fourier transform. Integr. Transf. Spec. Funct. 2011, 22, 477-486.

15. Shah, F.A. Construction of wavelet packets on p-adic field. Int. J. Wavelets Multiresolut. Inf. Process. 2009, 7, 553-565.

16. Shah, F.A.; Debnath, L. $p$-Wavelet frame packets on a half-line using the Walsh-Fourier transform. Integr. Transf. Spec. Funct. 2011, 22, 907-917.

17. Shah, F.A. p-Frame multiresolution analysis related to the Walsh functions. Int. J. Anal. Appl. 2015, 7, 1-15.

18. Shah, F.A. Tight wavelet frames generated by the Walsh polynomials. Int. J. Wavelets Multiresolut. Inf. Process. 2013, 11, 1350042. 
19. Golubov, B.I.; Efimov, A.V.; Skvortsov, V.A. Walsh Series and Transforms: Theory and Applications; Kluwer: Dordrecht, The Netherlands, 1991.

20. Schipp, F.; Wade, W.R.; Simon, P. Walsh Series: An Introduction to Dyadic Harmonic Analysis; Adam Hilger: Bristol, UK; New York, NY, USA, 1990.

(c) 2015 by the authors; licensee MDPI, Basel, Switzerland. This article is an open access article distributed under the terms and conditions of the Creative Commons Attribution license (http://creativecommons.org/licenses/by/4.0/). 